\title{
Adverse Drug Reactions in Ghanaian Children: Review of reports from 2000 to 2012 in VigiBase
}

\author{
Kennedy Obebi Cliff-Eribo ${ }^{1}$, Imti Choonara ${ }^{1}$, Alex Dodoo ${ }^{2}$, Delese M Darko ${ }^{2} \&$ Helen Sammons ${ }^{1}$ \\ 1 The University of Nottingham, Academic Division of Child Health, Derbyshire Children's \\ Hospital, Derby, United Kingdom \\ 2 WHO Collaborating Centre for Advocacy and Training in Pharmacovigilance, Accra, Ghana
}

\section{Objective}

To describe adverse drug reactions (ADRs) reported for children aged 0-17 years in Ghana.

\section{Methods}

Paediatric reports submitted by the Ghana National Centre for Pharmacovigilance to the World Health Organisation (WHO) Global ADR database, VigiBase up to December 2012 were extracted. The data was analysed for number of reports per year, types of reporters, and suspected ADRs and drugs.

\section{Results}

A total of 343 reports for children were received during the period. The drug classes most frequently reported were vaccines $(115,31 \%)$, antimalarials $(106,28 \%)$ and antibiotics $(57,15 \%)$. Nineteen of the top twenty individual drugs were anti-infectives. The most frequently reported ADRs were injection site infection, fever and rash. There were 23 deaths reported, and antimalarials were implicated in 12 cases.

\section{Conclusions}

Vaccines, antimalarials and antibiotics are the leading medicines reported to cause ADRs in Ghanaian children. There was a high mortality rate, with many of the deaths due to unexplained causes.

Keywords: adverse drug reactions, spontaneous reporting system, pharmacovigilance, children

\section{Introduction}

Adverse drug reactions (ADRs) have been recognised as a major global health concern ${ }_{[1]}$, even though overall reporting of ADRs across the world is generally low [2]. Due to developmental changes taking place during childhood, ADRs in children can be different to those in adults ${ }_{[3]}$. One in 10 children in hospital will experience an ADR ${ }_{[4]}$. The majority of studies of ADRs in children have been carried out in prospective observational settings on inpatients and outpatients. In the community, it was suggested that at least one in every 500 children experiences an ADR each year ${ }_{[5]}$.

Postmarketing surveillance of drugs by manufacturers plays a vital role in evaluating drug safety [6]. Programmes to promote the reporting of ADRs by healthcare professionals have been established by health authorities in many countries. These are supported by the World Health Organisation (WHO) Collaborating Centre for International Drug Monitoring, the Uppsala Monitoring Centre (UMC), Sweden $_{[7]}$ as well as WHO and its Collaborating Centres for pharmacovigilance (Ghana, Morocco, 
Netherlands). ADRs reported for children worldwide by member states of WHO International Drug Monitoring Programme have been analysed from VigiBase ${ }_{[8]}$. In addition, paediatric ADRs have been reported from national ADR databases of high-income countries in Europe ${ }_{[6,9]}, \mathrm{USA}_{[10]}$ and Cuba ${ }_{[5}$, 11]. However, there have been no published reports for children in Africa from a national pharmacovigilance database.

The population of Ghana is 25 million, of whom 11 million are children aged $0-17$ years. Ghana was the first country in West Africa to have a national pharmacovigilance (PV) centre. It joined the WHO Programme for International Drug Monitoring in 2001, and maintains an ADR database. The UMC and WHO then established centres in Ghana in 2009 (the then UMC-Africa as well as the WHO Collaborating Centre for Advocacy and Training in Pharmacovigilance), with the responsibility of promoting and supporting ADR reporting in Africa ${ }_{[12]}$. The national PV centre in Ghana is located within the national drug regulatory agency, the Ghana Food and Drugs Authority (FDA), with active regional centres throughout the country. The reports received are shared globally within the WHO Individual Case Safety Reports (ICSR) database, VigiBase ${ }_{[13]}$, managed by the UMC in Sweden. The aim of this study was to describe the type, frequency and severity of ADRs among Ghanaian children using this database.

\section{Methods}

The Ghana FDA gave approval for the use of the data, and consent from patients was not required. All the anonymised reports forwarded by the Ghana Food and Drugs Authority between 1999 and December 2012 to the International Drug Monitoring Centre, and entered into the WHO Global Individual Case Safety database, VigiBase were retrieved. The reports for children aged 0-17 years were identified and grouped into neonates, infants, children and adolescents according to ICH guidelines [14]. ADRs were described according to year, sex and age of the child, and notifier of reports. WHO-Adverse Reaction Terminology (WHO-ART) preferred and critical terms were also evaluated. WHO-ART critical terms are reported ADRs which may be indicative of serious disease states, and need quick and decisive action ${ }_{[15]}$. The suspect drugs were described according to WHODrug Dictionary (WHO-DD) preferred base name, and an individual ADR reported with more than one drug in a single case was considered only once.

\section{Results}

During the 14-year period, 343 reports containing 600 ADRs were received from a total of 1819 reports submitted to UMC by Ghana. This represented an average reporting rate of 2.5 reports per million children per year, and an average of 1.7 ADRs per report. There were no reports received in 1999 and 2001. In the year 2000, and between 2002 and 2007, the number ranged from one to eleven; thereafter, the number rose steadily from 24 in 2008 to a peak of 130 reports in 2010 (Figure 1).

For the cases where sex was indicated, female reports numbered 164 (48\%), and those for males were 167 (49\%). Children aged between 2-11 years made up just over a third of the reports (Table 1), as did reports for infants aged 28 days to 23 months.

Nurses submitted $172(50 \%)$ of the reports, pharmacists 79 (23\%), and physicians 52 (15\%). Information on reporter details on $40(12 \%)$ of the cases were not provided. 
A total of 601 ADRs were reported during the period (Table 2). Twenty-five percent of these were considered serious, including maculo-papular rash, which was linked to diclofenac. Injection site infection $(n=58)$, fever $(n=58)$, and rash $(n=34)$ were the most frequently reported ADRs. StevensJohnson syndrome was the sixth most frequently reported ADR, and was experienced by 27 children. According to system organ class, infants and children were reported with almost equal proportions of ADRs (35\% v $36 \%)$.

Vaccines, antimalarials, antibiotics and anthelmintics were the most frequently reported classes of drugs (Table 3). Vaccines (measles and pentavalent) were the predominant drug class for children 0 23 months, followed by antimalarials which were mostly reported for children aged 2-11 years.

Three hundred and seventy seven individual drugs were reported for the 343 children. Tetanus vaccine, measles vaccine, amodiaquine/artesunate and mebendazole were the individual drugs most frequently reported (Table 4). Six antimalarials, five antibiotics, four vaccines, and four anthelmintics were within the 20 medicines most frequently reported. Diclofenac was the only drug in the top 20 that was not an anti-infective agent.

There were 23 (6.7\%) fatalities recorded for the 343 reports. Anti-infective agents or vaccines were reported for all of the deaths (Table 5). Antimalarials were associated with 12 fatalities, antibiotics with 7 deaths, anthelmintics (4 deaths), and vaccines with 2 deaths. The antimalarial, quinine, was reported for 9 fatal cases. The reason for the fatalities was unknown in most cases, and in two cases, quinine was reported as ineffective. Stevens-Johnson syndrome was mostly associated with the reported deaths. Only one of the fatalities resulting from Stevens-Johnson syndrome was reported as certainly related to the drug, mebendazole. Causality assessments were recorded in 16 of the 23 fatalities. The majority were considered possible (Table 5).

An additional 61 children experienced a total of 64 non-fatal critical ADRs (Table 6). Stevens Johnson syndrome was the most frequent non-fatal critical ADR and was mainly reported with antibiotics and antimalarials.

\section{Discussion}

Underreporting has been globally acknowledged as a major challenge in pharmacovigilance practice [2], and the ADR reporting rate of 2.5 reports per million children per year recorded is thus not

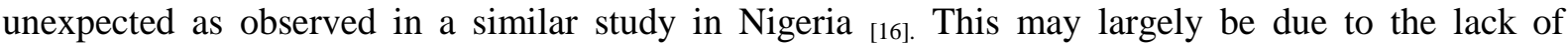
reporting forms, and awareness of ADR reporting procedures from healthcare professionals [17]. However, with the establishment of the WHO Collaborating Centre and the UMC office in Ghana in 2009 [18], awareness in pharmacovigilance has improved. The National Centre for Pharmacovigilance has also been active in its advocacy activities leading to increased reporting over the years though the fall in reports between 2011 and 2012 is of concern. Also of importance is the increasing pharmacovigilance activity of the FDA including the appointment of regional pharmacovigilance officers, and close collaboration between the FDA and the Expanded Programme on Immunisation in monitoring safety of vaccines.

The high number of nurses' reports observed may be attributed to their involvement in active monitoring of swine flu (H1N1) pandemic vaccination in Ghana ${ }_{[19]}$ and unique role in observing patients' early signs and symptoms of drug reactions ${ }_{[20]}$. In contrast, pharmacists in Canada ${ }_{[21]}$ and Netherlands [22] have been seen to report more ADRs for children, as patients consult with them directly at pharmacy outlets. The key to success in ADR reporting is adequate pharmacovigilance 
education for all healthcare professionals which has been adopted in Cuba, and improved reporting rates $[5]$.

A large proportion of reports in this study concerned children aged 2-11 years. This observation was similar, for the same group of children, to a study carried out in Spain ${ }_{[9]}$ but in contrast with the report from Denmark, where majority of cases reported were for infants aged $0-2$ years ${ }_{[6]}$. The large number of reports for children aged between 2-11 years probably relates to the prevalence of malaria and hence anti-malarials in this age group.

Anti-infectives, mainly vaccines constituted the highest number of medical product reported in this study. This observation was consistent with studies conducted in Sweden [23] and UK [24], and contrasted with reports in USA ${ }_{[10]}$ where medicines used to treat paediatric attention deficit hyperactivity disorder (ADHD) were the most frequently reported drugs. The interest shown by the national Expanded Programme on Immunisation (EPI) in safety monitoring, and its collaboration with the National Pharmacovigilance Centre, may have contributed to the relative large number of reports involving vaccines. The pentavalent vaccine was largely associated with fever and other injection site reactions in children aged between 0-2 months. These findings were consistent with previous studies conducted in Sweden and Cuba $[5,23]$.

Malaria is a major contributor of child mortality in sub-Saharan Africa, and chloroquine which was primarily used for its treatment was replaced with artesunate-amodiaquine as first line of therapy in

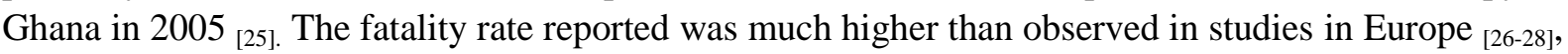
possibly due to (i) the high prevalence of malaria and hence the widespread use of antimalarials, (ii) reporting of mostly serious ADRs, or (iii) due to consumption of sub-standard medicines. The majority of the fatalities may be due to the disease (severe malaria) itself and not necessarily the antimalarials. In most of the cases, deaths from unknown cause and medicine ineffectiveness were associated with quinine. This suggests that the drugs could have been either counterfeit or sub-standard or the diagnosis inaccurate, and the fatalities reported may have resulted from the use of these possibly fake medicines or due to mis-diagnosis. Root cause analyses were not carried out thus it is impossible to tell the exact cause. The presence of falsified and sub-standard antimalarials, particularly, quinine in sub-Saharan Africa has been recognised [29], and in Ghana, sub-standard artemisinin-combination therapy have been sold in the open market [30]. The Ghana FDA has over the past 10 years taken active steps to improve detection of counterfeit medicines circulating in the country, and repeated surveys shows that the strong regulatory guidelines applied by the FDA are yielding results. Nonetheless, the FDA has placed measures to curb distribution of such hazardous medicines and alert consumers whenever they are identified $[31,32]$.

Three children who were administered the antibiotic, ceftriaxone, also died of an unknown cause. Unexplained sudden death with ceftriaxone has been reported by others in China ${ }_{[33]}$ and $\mathrm{Cuba}_{[5]}$.

Three of the 4 fatalities due to antihelminthics were recorded between 2008 and 2009. The first nationwide programme to deworm children in Ghana was initiated in 2007. However, the programme was derailed due to bad media publicity on rumours of death associated with the drugs used for the programme $[34,35]$. It is possible that these deaths may be linked to the programme or that the reporting of these cases was occasioned by the bad publicity.

Data incompleteness was a major limitation in this study. A dedicated committee of experts in pharmacovigilance (Technical Advisory/Expert Committee) regularly meet to assess the reports. However, most of the fatal reports did not contain causality assessment and other important information about the drugs such as dosage. This made interpretation of the results difficult. 


\section{Conclusion}

Vaccines, antimalarials and antibiotics are the leading medicines causing reported ADRs in Ghanaian children. There was a high mortality rate among the reports, compared to previous studies in European children. Of the 23 deaths reported, 6 were due to Stevens-Johnson syndrome which occurred following intake of pyrimethamine/sulfadoxine, mebendazole, albendazole and ivermectin.

\section{Acknowledgments}

We wish to acknowledge the Ghana Food and Drugs Authority (FDA) for approving the use of the data for this work. The support of the Petroleum Technology Development Fund (PTDF) is gratefully acknowledged.

The opinions and conclusions in this study are those of the authors, and do not represent those of the Ghana Pharmacovigilance Centre and WHO.

\section{Declaration of interest}

None 


\section{Bibliography}

Paper of special note have been highlighted as either of interest $(\cdot)$ or of considerable interest $(\cdot \cdot)$ to readers.

1. Pirmohamed, M., et al., Adverse drug reactions as cause of admission to hospital: prospective analysis of 18820 patients. BMJ, 2004. 329(7456): p. 15-9.

2. Hazell, L. and S.A. Shakir, Under-reporting of adverse drug reactions : a systematic review. Drug Saf, 2006. 29(5): p. 385-96.

3. Aagaard, L., A. Christensen, and E.H. Hansen, Information about adverse drug reactions reported in children: a qualitative review of empirical studies. Br J Clin Pharmacol, 2010. 70(4): p. 481-91.

4. Clavenna, A. and M. Bonati, Adverse drug reactions in childhood: a review of prospective studies and safety alerts. Arch Dis Child, 2009. 94(9): p. 724-8.

5. Barzaga Arencibia, Z., et al., Pharmacovigilance in children in Camaguey Province, Cuba. European Journal of Clinical Pharmacology, 2012. 68(7): p. 1079-1084.

This study is useful for comparison with similar studies in developing countries.

6. Aagaard, L., C.B. Weber, and E.H. Hansen, Adverse drug reactions in the paediatric population in Denmark: a retrospective analysis of reports made to the Danish Medicines Agency from 1998 to 2007. Drug Saf, 2010. 33(4): p. 327-39.

- This study is useful for comparison of paediatric ADRs across countries

7. Venulet, J. and M. Helling-Borda, WHO's International Drug Monitoring - The Formative Years, 1968-1975. Preparatory, Pilot and Early Operational Phases. Drug Saf, 2010. 33(7): p. e1-e23.

8. Star, K., et al., Suspected adverse drug reactions reported for children worldwide: an exploratory study using VigiBase. Drug Saf, 2011. 34(5): p. 415-28.

- This study is useful for comparison of paediatric ADRs across countries

9. Aldea, A., et al., Paediatric adverse drug reactions reported to the Spanish Pharmacovigilance System from 2004 to 2009. Eur J Clin Pharmacol, Sep;68(9):1329-38., 2012.

- This study is useful for comparison with similar studies in developing countries.

10. Johann-Liang, R., et al., Pediatric drug surveillance and the Food and Drug Administration's adverse event reporting system: an overview of reports, 2003-2007. Pharmacoepidemiol Drug Saf, 2009. 18(1): p. 24-7.

11. Arencibia, Z.B., et al., Adverse drug reactions in children in Camaguey Province, Cuba. Arch Dis Child, 2010. 95(6): p. 474-7.

This study is useful for comparison with similar studies in developing countries.

12. http://www.who-umc.org/. UMC - Africa. 12 November 2013]; Available from: http://www.who-umc.org/dynpage.aspx $? \mathrm{id}=98093 \& \mathrm{mn} 1=7347 \& \mathrm{mn} 2=7252 \& \mathrm{mn} 3$ $=7253 \& \mathrm{mn} 4=7333$. 
13. Lindquist, M., VigiBase, the WHO global ICSR database system: basic facts. Drug Information Journal, 2008. 42(5): p. 409-419.

14. EMEA. ICH Topic E 11. Clinical investigation of medicinal products in the paediatric population January 2001. CPMP/ICH/2711/99 200117 October 2013]; Available from: http://www.ema.europa.eu/docs/en_GB/document_ library/Scientific_guideline/2009/09/WC500002926.pdf.

15. http://www.umc-products.com. The Uppsala Monitoring Centre Critical Terms. 08 May 2014]; Available from: http://www.umc-products.com/DynPage.aspx?id=73559\&mn1= $1107 \& \mathrm{mn} 2=1664 \& \mathrm{mn} 3=6044$.

16. Cliff-Eribo, K., I. Choonara, and H. Sammons, G302 Adverse Drug Reactions in Nigerian Children. Archives of Disease in Childhood, 2014. 99(Suppl 1): p. A124-A124.

This study is useful for comparison of paediatric ADRs across countries

17. Sabblah, G., et al., Adverse drug reaction reporting by doctors in a developing country: a case study from ghana. Ghana Med J, 2014. 48(4): p. 189-93.

.. $\quad$ This is an essential overview of ADR reporting by healthcare professionals in developing countries.

18. UMC - Africa. What we do? 25 September 2014]; Available from: http://www.whoumcafrica.org/index.php/what-we-do.

19. Nzussouo, N.T., et al., Delayed 2009 pandemic influenza A virus subtype H1N1 circulation in West Africa, May 2009-April 2010. J Infect Dis, 2012. 206 Suppl 1: p. S101-7.

20. Backstrom, M., E. Ekman, and T. Mjorndal, Adverse drug reaction reporting by nurses in Sweden. Eur J Clin Pharmacol, 2007. 63(6): p. 613-8.

21. Carleton, B.C., et al., Paediatric adverse drug reaction reporting: understanding and future directions. Can J Clin Pharmacol, 2007. 14(1): p. e45-57.

22. Schirm, E., et al., Reported adverse drug reactions and their determinants in Dutch children outside the hospital. Pharmacoepidemiol Drug Saf, 2004. 13(3): p. 159-65.

23. Kimland, E., et al., Paediatric adverse drug reactions reported in Sweden from 1987 to 2001. Pharmacoepidemiol Drug Saf, 2005. 14(7): p. 493-9.

24. Hawcutt, D.B., et al., Reported paediatric adverse drug reactions in the UK 2000-2009. Br J Clin Pharmacol, 2012. 73(3): p. 437-46.

- This study is useful for comparison of paediatric ADRs across countries

25. Dodoo, A.N., et al., Pattern of drug utilization for treatment of uncomplicated malaria in urban Ghana following national treatment policy change to artemisinin-combination therapy. Malar J, 2009. 8: p. 2.

26. Marques, J., et al., A survey of spontaneous reporting of adverse drug reactions in 10 years of activity in a pharmacovigilance centre in Portugal. Int J Pharm Pract, 2014. 22(4): p. 275-82. 
27. Motola, D., et al., Ten years of pharmacovigilance in Italy: the experience of Emilia-Romagna region in the monitoring of drug's safety profile. Expert Opin Drug Saf, 2014. 13(7): p. 86773.

28. McLernon, D.J., et al., Adverse drug reaction reporting in the UK: A retrospective observational comparison of yellow card reports submitted by patients and healthcare professionals. Drug Safety, 2010. 33(9): p. 775-788.

29. Nayyar, G.M., et al., Poor-quality antimalarial drugs in southeast Asia and sub-Saharan Africa. Lancet Infect Dis, 2012. 12(6): p. 488-96.

30. El-Duah, M. and K. Ofori-Kwakye, Substandard artemisinin-based antimalarial medicines in licensed retail pharmaceutical outlets in Ghana. J Vector Borne Dis, 2012. 49(3): p. 131-9.

31. http://www.ghana.gov.gh. FDA Cautious Public On Fake Antimalarial 03 June 2014]; Available from: http://www.ghana.gov.gh/index.php/2012-02-08-08-32-47/general-news/ 4063-fda-cautious-public-on-fake-antimalarial.

32. http://www.fdaghana.gov.gh/. Current Press Releases 13th September 2013 PUBLIC HEALTH WARNING: AVOID PATRONISING GSUNATE PLUS SUPPOSITORY. 10 JUNE 2015]; Available from: http://www.fdaghana.gov.gh/index.php?option=com_content\&view $=$ article \&id $=26 \&$ Itemid $=4$.

33. Yao, Y., R. Zhou, and Y. Wang, Fatal adverse effects of injected ceftriaxone sodium in China. Pharmacoepidemiol Drug Saf, 2012. 21(11): p. 1197-201.

34. Dodoo, A., et al., When rumours derail a mass deworming exercise. Lancet, 2007. 370(9586): p. 465-6.

35. Hall, A., et al., A review and meta-analysis of the impact of intestinal worms on child growth and nutrition. Maternal \& child nutrition, 2008. 4(s1): p. 118-236. 
Table 1: Age distribution of reports and adverse drug reactions (ADRs)

\begin{tabular}{lrr}
\hline Age group & n & Reports \\
& 5 & $\%$ \\
\hline Neonates $\leq 27$ days & 113 & 1 \\
28 days to 23 months & 133 & 33 \\
$2-11$ years & 92 & 39 \\
$12-17$ years & 343 & 27 \\
Total & & 100 \\
\hline
\end{tabular}

Table 2: Most frequently reported ADRs (preferred terms)

\begin{tabular}{lrr}
\hline ADR (preferred terms) & n & \% \\
\hline Injection site infection & 58 & 10 \\
Fever & 50 & 8 \\
Rash & 34 & 6 \\
Pruritus & 28 & 4 \\
Vomiting & 28 & 4 \\
Stevens Johnson syndrome & 27 & 4 \\
Abdominal pain & 21 & 4 \\
Asthenia & 18 & 3 \\
Skin reaction localised & 17 & 3 \\
Urticaria & 16 & 3 \\
Rigors & 15 & 2 \\
Diarrhoea & 13 & 2 \\
Convulsions & 11 & 2 \\
Dizziness & 11 & 2 \\
Conjunctivitis & 10 & 2 \\
Others* & 244 & 41 \\
Total & 601 & 100 \\
\hline
\end{tabular}

*ADRs with 9 or less reports were grouped together as others. Serious ADRs are in bold 
Table 3: Drug classes

\begin{tabular}{lrr}
\hline Medicine class & $\mathbf{n}$ & $\mathbf{\%}$ \\
\hline Vaccines & 115 & 30.5 \\
Antimalarials & 106 & 28.1 \\
Antibiotics & 57 & 15.1 \\
Anthelmintics & 53 & 14.1 \\
Vitamins & 9 & 2.4 \\
Anti-inflammatory & 8 & 2.1 \\
Anti-epileptics & 5 & 1.3 \\
Immunoglobulins & 5 & 1.3 \\
Analgesics & 4 & 1.0 \\
Antivirals & 3 & 0.8 \\
Anaesthetics & 2 & 0.5 \\
Antihistamines & 2 & 0.5 \\
Herbal products & 2 & 0.5 \\
Others & 6 & 1.8 \\
Total & 377 & 100 \\
\hline
\end{tabular}

Table 4: Individual drugs and frequency of reports

\begin{tabular}{lr}
\hline Drugs & $\begin{array}{r}\text { Number reports } \\
(\mathbf{n})\end{array}$ \\
\hline Tetanus vaccine & 56 \\
Measles vaccine & 37 \\
Amodiaquine/artesunate & 28 \\
Mebendazole & 25 \\
Amodiaquine & 21 \\
Ivermectin & 20 \\
Quinine & 19 \\
Pyrimethamine/sulfadoxine & 13 \\
Ceftriaxone & 10 \\
Others & 9 and below \\
\hline
\end{tabular}


Table 5: Death reports and suspected medicines and ADRs

\begin{tabular}{lrl}
\hline ADRs & Number & Suspect drugs \\
\hline Stevens Johnson syndrome & 5 & Benzylpenicillin (1), Pyrimethamine/sulfadoxine (2), \\
& & Flucloxacillin (1), Mebendazole (1) \\
Stevens Johnson syndrome, acute renal & 1 & Albendazole and Ivermectin (1) \\
failure and sepsis & & \\
Unknown* & 9 & Quinine (5), Quinine and Ceftriaxone (2), Ceftriaxone (1), \\
& & Chloroquine (1) \\
Medicine ineffective & 2 & Quinine (2) \\
Diarrhoea & 1 & Ivermectin (1) \\
Hyperpyrexia & 1 & Ivermectin (1) \\
Erythema multiforme & 1 & Metronidazole (1) \\
Convulsion & 1 & Cefuroxime (1) \\
Uncertain** & 2 & Tetanus vaccine (1), Measles vaccine (1) \\
\hline
\end{tabular}

*Death was recorded as the ADR in these cases.

**Recorded with injection site bleeding, and agitation, constipation, dyspnoea, increased sweating and vomiting, but cause of death unknown.

Table 6: WHO-ART critical ADRs and suspect drugs

\begin{tabular}{|c|c|c|}
\hline Critical ADRs & Number & Suspect drugs \\
\hline $\begin{array}{l}\text { Stevens Johnson } \\
\text { syndrome }\end{array}$ & 21 & $\begin{array}{l}\text { Antibiotics (7), Antimalarials (5), Anthelmintics (3), Vaccines (3), } \\
\text { Anti-convulsants (2), Vitamins and blood products (1) }\end{array}$ \\
\hline Dystonia & 9 & Antimalarials (8), Propulsives (1) \\
\hline Face oedema & 9 & Antimalarials (4), Anthelmintics (2), Antibiotics (2), Immunoglobulins (1) \\
\hline Hyperpyrexia & 8 & Antimalarials (7), Anthelmintics (1) \\
\hline Mouth oedema & 4 & Antimalarials (2), Antibiotics (2) \\
\hline Dyskinesia & 3 & Antimalarials (3) \\
\hline Neonatal jaundice & 3 & Vitamins (3) \\
\hline Anaphylactic reaction & 2 & Anthelmintics (2) \\
\hline Erythema multiforme & 2 & Antimalarials (1), Antibiotics (1) \\
\hline Generalised oedema & 2 & Antibiotics (1), Vitamins (1) \\
\hline Paralysis flaccid & 2 & Vaccines (2) \\
\hline
\end{tabular}


Figure 1: Number of reports by year

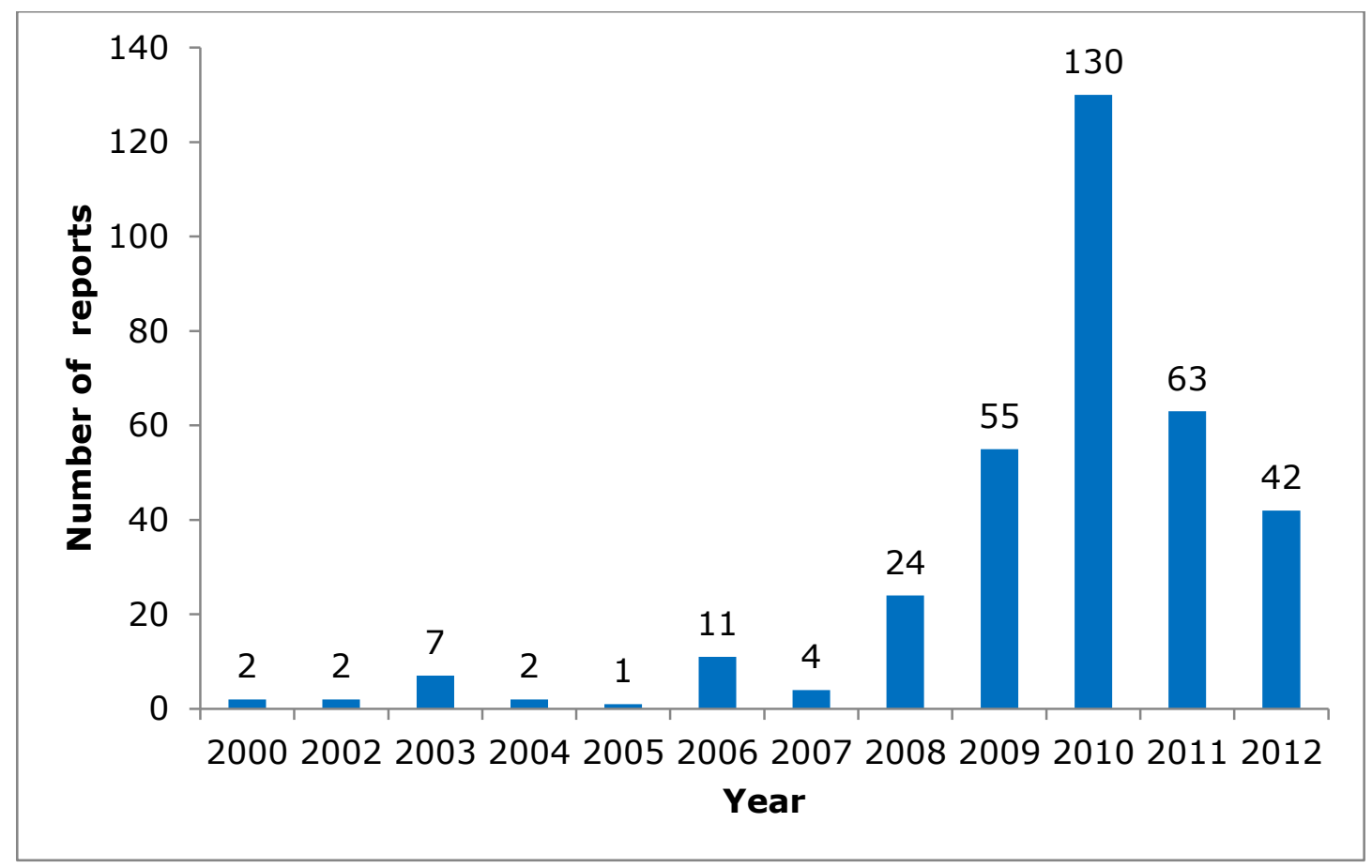

